We wish to express our thanks to the Radiation Laboratory of the University of California for the gift of molybdenum. A more detailed account will be published in the Rend. Lincei.

C. Perrier.

E. Segrì.

Royal University,

Palermo.

June 13.

'Geilmann and Weibke, Z. anorg. allgem. Chemie, 199, 120 (1931).

\section{Optical Rotatory Power of Turbid Solutions in an Electric Field}

IN a previous note in this journal ${ }^{1}$, A. McLean and I published the results of some experiments on the rotation of the plane of polarization of organic compounds dissolved in various solvents under the action of an electric field. These measurements were continued, and the results published in the Philosophical Magazine ${ }^{2}$ by J. Kunz and P. H. Babcock.

For some solutions the results could be repeated, but for other solutions the curves obtained were very complicated and could not be repeated, so that the explanation of the phenomenon given became doubtful. These measurements were continued, and we found that the solution of methylester in benzene showed a slight turbidity, due probably to a suspension of naphthalic anhydride; indeed the carefully filtered solution gave no effect, even under the action of alternating fields so high as 15,000 volts per centimetre. Therefore, either some type of compensation is present in this particular solution (such as opposite effects on solvent and ester), or the magnitude of rotation does not depend so much upon the electrical structure as was first assumed. As in the present experiment, the expected effect was not observed, the dipole induced in the given fields may be too small.

When it was found that the rotation was due to a suspension, experiments were carried out using finely grounded calcite, quartz, iron oxide $\left(\mathrm{Fe}_{2} \mathrm{O}_{3}\right)$, china clay, sodium chloride, etc., all of which gave an effect. The calcite suspension in benzene was one of particular interest, since it gave rotations from zero to $15^{\circ}$; in some cases a maximum rotation of $5^{\circ}$ was observed at about 3,000 volts per centimetre; then the rotation decreased with increasing fields, reached a minimum and began to increase again. When the field decreased, the curve was either above or below the branch obtained by increasing fields. These features depended upon the platinum electrodes in the polarimeter tube, which were too flexible and were moved upon application of the higher voltages. The difference in the two paths is due to settling or to mixing. The tube was reconstructed by supporting the electrodes in three places, and then the rotation reached a constant value at comparatively low fields.

The optical activity of suspensions is well known; it has received much attention by French and German physicists and chemists ${ }^{3}$.

University of Illinois,

J. Kunz.

R. G. LaBaw.

Urbana, Illinois.

June 5.

1 Nature, 136, 795 (1935).

${ }^{2}$ Phil. Mag., (7), 22, 616 (October 1936).

'See, for example, Stefan Procopiu, Ann. Phys., 1, 213 (1924).
WE have been investigating the effect described by Prof. Kunz and R. G. LaBaw, first in conjunction with McLean, who, however, had to leave it to take up other work. Although the solutions were apparently quite clear and free from obvious particles, we were led to suspect in some cases that the result might be due to very minute crystals in suspension in the liquid; we have also heard from Prof. Kunz that he has come to the same conclusion. Very careful filtration through selected filter papers removes the optical activity. The conclusion is, then, that the effect is due to dichroism induced in the particles by the electric field, a phenomenon not entirely new, although its accurate quantitative investigation is beset by numerous difficulties.

E. B. Ludlam.

A. W. Pryde

H. Gordon Rule.

Chemistry Department,

University, Edinburgh. June 16.

\section{Air-drag and the Equilibrium. of Whirling Threads}

IN the absence of all resistance to motion and assuming perfect flexibility, the differential equations of motion for a thread which carries a small spherical 'bob' and is whirled rapidly about a vertical axis by a horizontal arm give us an approximate solution for small amplitudes a plane sine wave-with reference to moving axes-leading to the well-known formula for plane vibrations ${ }^{1}$. This approximate solution throws no light whatever on the stability of whirling threads as observed experimentally in air: these threads show 'necks' of appreciable amplitude in place of point nodes, the necks increasing in diameter the farther they are from the 'bob'. With sufficiently long threads and a large radius of the whirling arm, the sine outline disappears altogether.

I have recently found that if a term be introduced for air-drag-assumed proportional to the square of the linear velocity - the differential equations then admit of an approximate solution which not only gives the outline of the thread with surprising accuracy but also fully explains the limited stability of the system as described by Hall (loc. cit.).

For ease in experimental interpretation, the solution is best referred to moving rectangular axes, the vertical axis of whirl being the $Z$-axis. The form of solution is then :

$x=R\left(1+\frac{1}{3} \mu^{3} z^{3}+\frac{1}{18} \mu^{6} z^{6}\right.$. . . $) \sin \beta z$,

$y=R \mu z\left(1+\frac{1}{6} \mu^{3} z^{3}+\frac{2}{6} \mu^{6} z^{6} \ldots\right) \quad \cos \beta\left(1+\frac{1}{4} R^{2} \beta^{2}\right) z$;

where $z$ is measured from the node of the lowest balloon, $R$ is, effectively, the radius of the lowest balloon, determined by the radius of the whirling arm; $\beta^{2}$ is $m \omega^{2} / M g ; m$ is thread mass per centi. metre ; $\omega$ is angular velocity of whirl ; $M$ is mass of 'bob'; $g$ is gravitational constant; $\mu$ is $(\gamma / 2 \beta)\left(1-\frac{1}{6} R^{2} \beta^{2}\right) R ; \gamma$ is $P \omega^{2} / M g ; P$ is coefficient of air-drag per centimetre of vertical thread.

This solution gives the projection of the spiral thread on two perpendicular vertical planes. The $x$-values are conveniently referred to as the 'centrifugal wave', the $y$-values as the 'drag wave'. The period of the drag wave differs from that of the centrifugal wave by the small factor $\frac{1}{4} R^{2} \beta^{2}$, so that the positions of minimum amplitude do not coincide exactly with the nodes of the centrifugal wave. This point is clearly shown in photograph $b$ of Hall's 